\title{
Correlation Length Bounds for Disordered Ising Ferromagnets
}

\author{
J. T. Chayes ${ }^{1, \star}$, L. Chayes ${ }^{1, \star}$, Daniel S. Fisher ${ }^{2, \star \star}$, and T. Spencer ${ }^{3, \star \star \star}$ \\ ${ }^{1}$ Department of Mathematics, University of California, Los Angeles, CA 90024, USA \\ ${ }^{2}$ Department of Physics, Princeton University, Princeton, NJ 08544, USA \\ ${ }^{3}$ The Institute for Advanced Study, Princeton, NJ 08540, USA
}

\begin{abstract}
The $d$-dimensional, nearest-neighbor disordered Ising ferromagnet:

$$
\mathscr{H}=-\sum J_{i j} \sigma_{i} \sigma_{j}
$$

is studied as a function of both temperature, $T$, and a disorder parameter, $\lambda$, which measures the size of fluctuations of couplings $J_{i j} \geqq 0$. A finite-size scaling correlation length, $\xi_{f}(T, \lambda)$, is defined in terms of the magnetic response of finite samples. This correlation length is shown to be equivalent, in the scaling sense, to the quenched average correlation length $\xi(T, \lambda)$, defined as the asymptotic decay rate of the quenched average two-point function. Furthermore, the magnetic response criterion which defines $\xi_{f}$ is shown to have a scale-invariant property at the critical point. The above results enable us to prove that the quenched correlation length satisfies:
\end{abstract}

$$
C|\log \xi(T)| \xi(T) \geqq\left|T-T_{c}\right|^{-2 / d},
$$

which implies the bound $v \geqq 2 / d$ for the quenched correlation length exponent.

\section{Introduction}

The behavior of the correlation length exponent for disordered systems has been a subject of interest for some time. In 1974, Harris [2] suggested a simple criterion to determine whether or not the critical behavior of a given system is affected by a small amount of disorder. He argued that if the correlation length exponent, $v_{p}$, of a $d$-dimensional pure (uniform) system satisfies the bound $v_{p}>2 / d$, then disorder is irrelevant in the renormalization group sense and thus should not change the critical behavior. A natural generalization of Harris' result (which does not, however, follow directly from his line of argument) is that in all disordered systems with continuous transitions, the correlation length exponent ought to satisfy the bound

$$
v \geqq \frac{2}{d} .
$$

$\star$ Work supported in part by National Science Foundation Postdoctoral Fellowships

$\star \star$ Work supported in part by National Science Foundation Grant No. DMR-87-19523

$\star \star \star$ Work supported in part by National Science Foundation Grant No. DMR-84-01225 
This should hold independent of whether or not the critical behavior is the same as in the uniform system, and indeed even when the system does not have a uniform analogue (e.g., disordered electronic systems [3]). However, the general applicability of the bound (1.1) does not appear to have been widely anticipated.

A number of works on random exchange Ising magnets have supported (1.1). Calculations in both two [4] and higher [5] dimensions yield results satisfying (1.1); in $d=2$ the bound is saturated. An appropriate modification of (1.1) for magnets with long-range correlated bond disorder was derived by Weinrib and Halperin [6].

Recently [1], we considered a broad class of disordered systems with independent randomness characterized by some disorder (e.g., impurity concentration) parameter, $\lambda$. We defined a probabilistic finite-size scaling correlation length, $\xi_{f}$, and proved that when the system passes through a critical value, $\lambda_{c}$, of the disorder parameter - as signaled by the divergence of $\xi_{f}$ - then $\xi_{f}$ $\geqq$ (const) $\left|\lambda-\lambda_{c}\right|^{-2 / d}$. This implies the bound (1.1) for the exponent $v$.

The conditions of the theorem in [1] allow a broad spectrum of choices from which one may select (or construct) a correlation length $\xi_{f}$; furthermore, if one has any faith in universality, the particular choice of $\xi_{f}$ should be unimportant. However, from the mathematical point of view, this faith requires substantiation. Indeed, most systems have a natural, intrinsic correlation length $\xi$, typically given by the asymptotics of an appropriate two-point function. In order to prove that the correlation length exponent associated with $\xi$ satisfies the bound (1.1), it is sufficient to show that

(a) there is a critical point $\lambda_{c}$ at which some $\xi_{f}$ diverges continuously, and

(b) $C|\log \xi| \xi \geqq \xi_{f}$ as $\lambda \uparrow \lambda_{c}$.

Unfortunately, at present, the only system in which this has been completely verified is $d$-dimensional short-range percolation (for which it follows from Proposition 3.2 of [7]).

The principal purpose of this paper is to extend the more complete analysis to disordered Ising ferromagnets. Thus we consider systems specified by the (formal) Hamiltonian

$$
\mathscr{H}=-\sum_{\langle i, j\rangle} J_{i j} \sigma_{i} \sigma_{j},
$$

where the sum is over all nearest-neighbor pairs (bonds) of the $d$-dimensional hypercubic lattice, $\sigma_{i}= \pm 1$ are the usual Ising spins, and the ferromagnetic couplings $J_{i j} \geqq 0$ are i.i.d. variables with distribution $\varrho$. We will treat a rather general class of distributions, which includes in particular the physically interesting case of dilute ferromagnets. (For a precise specification of the allowed $\varrho$, see Sect. 3.)

An intrinsic correlation length for the disordered Ising ferromagnet is the quenched correlation length, $\xi$, defined in the high-temperature phase as the exponential decay rate of the quenched two-point function: informally

$$
\overline{\left\langle\sigma_{0} \sigma_{x}\right\rangle} \sim e^{-|x| / \xi},
$$

where \langle\rangle denotes the thermal expectation, and the overbar denotes quenched averaging - i.e., averaging with respect to the bond disorder distribution. Thus $\xi^{-1}$ 
$=-\lim _{x \rightarrow \infty}|x|^{-1} \log \overline{\left\langle\sigma_{0} \sigma_{x}\right\rangle}$. A priori there is an alternative intrinsic correlation

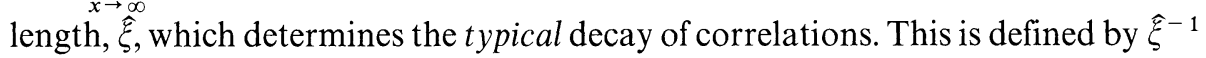
$=-\lim _{x \rightarrow \infty}|x|^{-1} \log \left\langle\sigma_{0} \sigma_{x}\right\rangle$, with probability one. While it is clear that $\xi \geqq \hat{\xi}$, it is not known under what circumstances the scaling behavior of $\xi$ and $\hat{\xi}$ are the same. However, it is the quenched averaged correlation function (1.3) which is measured in scattering experiments, and therefore $\xi$ is the relevant correlation length. Henceforth, we will take (1.3) as our definition of the intrinsic correlation length.

As mentioned above, there are many possible definitions of a finite-size scaling correlation length. For disordered Ising ferromagnets, we construct one such length, $\xi_{f}$, in terms of an event which measures the magnetic response of large cubes. The crux of this paper (Sect. 4 ) is a proof that $\xi_{f}$ is equivalent, in the scaling sense, to the fundamental correlation length $\xi$ :

$$
\frac{\xi_{f}}{c_{1} \log \xi_{f}+c_{2}} \leqq \xi \leqq c_{3} \xi_{f}+c_{4}
$$

where the $c_{i}$ are positive, finite constants. Given this equivalence, we establish, via finite-volume continuity, the existence of a bona fide critical point, i.e., a point where the correlation length diverges continuously. The inequality (1.1) can then be obtained for the fundamental $v$ from the lower bound in (1.4) and the theorem of [1].

The rest of this introduction is devoted to possible extensions of (1.1), and the remainder of the paper is organized as follows:

In Sect. 2, we will briefly review our previous theorem. The proof given in [1] was for systems with disorder distributions of a percolation-like form, in which the phase transition is approached by varying the disorder parameter, holding other parameters fixed. However, as was mentioned in [1], the result also applies to systems with fixed smooth disorder distributions, in which the phase transition is approached by varying an additional parameter (e.g. temperature in disordered magnets or energy in disordered electronic systems). The extension to these cases is not difficult: the details are provided in Sect. 2.

In Sect. 3, we will give precise definitions of random Ising ferromagnets, derive some basic (infinite-volume) properties of these systems, and define, for the single phase regime, the quenched correlation length.

In Sect. 4, we will construct our finite-size scaling event, and establish equivalence of the resulting length scale and the quenched correlation length. We will then prove criticality of the transition, from which the bound (1.1) follows. The more technical aspects of the proof of the equivalence between $\xi_{f}$ and $\xi$ are relegated to an appendix.

1i. Extensions to Systems with Correlated Disorder. Weinrib and Halperin [6] have considered the effects of correlations in the disorder, i.e., for the case of ferromagnets, in the $\left\{J_{i j}\right\}$. Short-ranged correlations are not expected to affect the results, provided the transition remains continuous. However, if the correlations are long enough ranged that the variations in the $\left\{J_{i j}\right\}$ over cubes of size $L$ are of order $L^{-\hat{d} / 2}$ for large $L$, with $\hat{d}<d$, then stronger inequalities than (1.1) should hold. 
In particular, we expect [6]

$$
v \geqq \frac{2}{\hat{d}}
$$

Indeed, Weinrib and Halperin [6] argue that for positive correlations of the $\left\{J_{i j}\right\}$ decaying as a power of $|i-j|$, Eq. (1.5) should hold as an equality. Extensions of our rigorous results for finite-size scaling correlation lengths to this case may well be possible.

Another interesting case is when the couplings depend not on all $d$ coordinates, but only on a subset $d_{R}<d$ of the coordinates, while the system is uniform along $d-d_{R}$ dimensional hyperplanes. For disordered Ising ferromagnets, such systems turn out to be rather pathological. Nevertheless, if a continuous transition occurs with a diverging finite-size scaling correlation length in the random directions, $\xi_{f R}$, the natural expectation is that the associated exponent will satisfy

$$
v \geqq \frac{2}{d_{R}} .
$$

Exact results for the random Ising ferromagnet with $d=2$ and $d_{R}=1$ have been obtained $[8,9]$. In particular, the exponent of the typical correlation length is found not to satisfy Eq. (1.6). However, as discussed in [10], the quenched correlation length seems to satisfy the bound (1.6) as an equality. Although this model is rather peculiar, and its behavior is not yet entirely understood, one certainly should be cautious in attempting to infer too general conclusions about relations between various possible correlation lengths from the results of this paper.

\section{Correlation Length Bounds for General Disordered Systems}

Here we review and slightly extend the results of [1]. The reader is referred to the original reference for more details and, particularly, for explicit examples.

The generality of the result in [1] followed from noting that, independent of the specific properties of a particular disordered system, the underlying randomness could be introduced in such a way as to define a percolation-like problem. Then if we examine only the behavior of the sample configurations (e.g., as a function of the disorder parameter), certain natural questions arise analogous to those for the percolation transition. In particular, in the context of disordered systems undergoing continuous transitions, one is immediately led to define length scales within which events characterizing the critical behavior are likely to occur and beyond which they will be rarely observed. At the critical point, some such events should be likely on all scales. This suggests that the largest scale at which such an event typically occurs should be identified with the correlation length of the system.

To make explicit the connection between a general disordered system and percolation, we took the disorder distribution to be describable as a convex combination of two distributions:

$$
\varrho_{\lambda}=\lambda \varrho_{1}+(1-\lambda) \varrho_{2} .
$$


Thus the disorder parameter $\lambda$ is analogous to a bond or site density in percolation. For example, in disordered Ising ferromagnets, $\varrho_{1}$ and $\varrho_{2}$ could simply be $\delta$-functions at distinct couplings. However, in general, $\varrho_{1}$ and $\varrho_{2}$ need not describe pure systems, so that even the points $\lambda=0$ or $\lambda=1$ may be genuine disordered systems. Nonetheless, it was supposed that a critical transition occurred at some $\lambda_{c}$ with

$$
0<\lambda_{c}<1 .
$$

It was further supposed that the transition could be characterized by a sequence of events, $\left(\mathscr{A}_{L}\right)$, occurring in volumes of scale $L$ such that:

at $\lambda_{c}$, on all scales,

$$
\operatorname{Prob}_{\lambda_{c}}\left(\mathscr{A}_{L}\right) \geqq c
$$

while for $\lambda<\lambda_{c}$,

$$
\lim _{L \rightarrow \infty} \operatorname{Prob}_{\lambda}\left(\mathscr{A}_{L}\right)=0 \text {. }
$$

Thus

$$
\xi_{f}(\lambda) \equiv \max \left\{L \mid \operatorname{Prob}_{\lambda}\left(\mathscr{A}_{L}\right) \geqq \frac{1}{2} c\right\}
$$

may be identified as a finite-size scaling correlation length. The result of [1] is:

Theorem 2.1 [1]. Under the conditions (2.1)-(2.4),

$$
\xi_{f}(\lambda) \geqq C\left|\lambda-\lambda_{c}\right|^{-2 / d}
$$

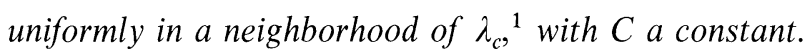

Given the conventional definition: $\xi_{f}(\lambda) \sim\left|\lambda-\lambda_{c}\right|^{-v}$, this is the bound $v \geqq \frac{2}{d}$.

Note that although the above theorem is rather general, it does require that:(1) the disorder distribution be expressible as a convex combination of two other distributions; and (2) the phase transition be approached by varying the disorder (convex mixing) parameter. Although, by universality, one would argue that neither of these restrictions should change the nature of the critical point (and, in particular, the bound on $v$ ), an explicit demonstration of this claim may be useful.

Now suppose we have a disordered system with a fixed smooth disorder distribution, $\varrho$, and that we wish to approach the critical point by varying an additional parameter (e.g. the temperature in disordered magnets or the Fermi energy in disordered electronic systems). For brevity of notation (at the possible expense of confusing the reader), we will call this additional parameter $\lambda$, although here $\lambda$ is to be though of as a temperature or energy variable. Then, given the disorder distribution, $\varrho(x)$, we can define a new distribution, $\varrho_{\lambda}(x)$, which scales with the additional parameter $\lambda$. In disordered magnets, where the couplings are multiplied by the inverse temperature $\beta$, the appropriate scaling is multiplicative: $\varrho_{\lambda}(x) \equiv \lambda \varrho(\lambda x)$. In disordered electronic systems, where the random potentials are determined up to an additive energy factor (the chemical potential), the distribution should be scaled additively: $\varrho_{\lambda}(x) \equiv \varrho(x+\lambda)$.

\footnotetext{
${ }^{1}$ In cases where (2.3) only holds for a sparse sequence of lengths, (2.6) holds on a sequence of $\lambda$ 's converging to $\lambda_{c}$. One still gets $v \geqq 2 / d$, where $v$ is defined via a superior limit
} 
In either case, we again expect that there should be a length scale characterizing the transition, and that this length scale should be expressible in terms of the probability - with respect to the scaled distribution $\varrho_{\lambda}-$ of a sequence of finite-size scaling events. Thus we will again assume (2.3)-(2.4), which thereby allows us to define the finite-size scaling correlation lengths (2.5). Now, however, rather than requiring a disorder distribution of the form (2.1) and the existence of a non-trivial critical point (2.2), we require only a smoothness condition on the (unscaled) disorder distribution: Explicitly, we suppose that

$$
M_{\varrho} \equiv \int x^{2} \frac{\left[\varrho^{\prime}(x)\right]^{2}}{\varrho(x)} d x<\infty
$$

for multiplicative scaling, or

$$
A_{\varrho} \equiv \int \frac{\left[\varrho^{\prime}(x)\right]^{2}}{\varrho(x)} d x<\infty
$$

for additive scaling. We have

Theorem 2.2. Suppose the disorder distribution $\varrho \in C^{2}$ is a density supported on all of $\mathbb{R}$ or all of $\mathbb{R}^{+}$, and that it satisfies either condition $(2.7 \mathrm{M})$ or condition $(2.7 \mathrm{~A})$. Further suppose that there is a sequence of events $\left(\mathscr{A}_{L}\right)$ such that (2.3)-(2.4) hold with respect to the multiplicatively or additively scaled distribution: $\varrho_{\lambda}(x)=\lambda \varrho(\lambda x)$ or $\varrho_{\lambda}(x)=\varrho(x+\lambda)$. Then the conclusion of Theorem 2.1 holds.

Theorem 2.2 is an easy consequence of the following lemma.

Lemma 2.3. Suppose $\varrho \in C^{2}$ is a probability density supported on all of $\mathbb{R}$ or all of $\mathbb{R}^{+}$, satisfying $(2.7 \mathrm{M})$ or $(2.7 \mathrm{~A})$. Let $X_{1}, \ldots, X_{N}$ be random variables independently distributed according to $\varrho$, and let $F\left(X_{1}, \ldots, X_{N}\right)$ be a bounded function of these variables. Let $\varrho_{\lambda}(x)$ be a one-parameter family of scaled densities: either $\varrho_{\lambda}(x)$ $=\lambda \varrho(\lambda x)$ or $\varrho_{\lambda}(x)=\varrho(x+\lambda)$, and let $E_{\lambda}$ denote the corresponding expectation. Then

$$
\left|\frac{d E_{\lambda}(F)}{d \lambda}\right| \leqq\left[(\max F) \lambda^{-1} \sqrt{3+I_{\varrho}}\right] \sqrt{N},
$$

where $I_{\varrho} \equiv M_{\varrho}$ or $I_{\varrho} \equiv A_{\varrho}$ as given by (2.7).

Proof of Theorem 2.2 (given Lemma 2.3). According to the proof of Theorem 2.1 in [1], the key ingredient is to show that if $Y$ is an event depending on $N$ i.i.d. variables with distribution parameterized by $\lambda$, then $(d / d \lambda) \operatorname{Prob}_{\lambda}(Y) \leqq \alpha \sqrt{N}$ with $\alpha=\alpha(\lambda)$ e.g. continuous in $\lambda$. Thus we simply take $F$ in Lemma 2.3 to be the indicator function of the event $Y$.

Proof of Lemma 2.3. For simplicity, we will treat only the multiplicative case; the additive case follows from an identical argument. We write

$$
E_{\lambda}(F)=\int F\left(x_{1}, \ldots, x_{N}\right) \prod_{j=1}^{N} \varrho_{\lambda}\left(x_{j}\right) d^{N} x
$$


so that

$$
\begin{aligned}
\frac{d E_{\lambda}(F)}{d \lambda} & =\int F\left(x_{1}, \ldots, x_{N}\right) \sum_{k} \frac{d}{d \lambda} \varrho_{\lambda}\left(x_{k}\right) \prod_{j \neq k}^{N} \varrho_{\lambda}\left(x_{j}\right) d^{N} x \\
& =\int F\left(x_{1}, \ldots, x_{N}\right) \sum_{k}\left[\frac{1}{\lambda}+\frac{x_{k} \varrho_{\lambda}^{\prime}\left(x_{k}\right)}{\varrho_{\lambda}\left(x_{k}\right)}\right] \prod_{j} \varrho_{\lambda}\left(x_{j}\right) d^{N} x
\end{aligned}
$$

Taking absolute values,

$$
\begin{aligned}
\left|\frac{d E_{\lambda}(F)}{d \lambda}\right| & \leqq(\max F) \frac{1}{\lambda} \int\left|\sum_{k} 1+\frac{\lambda x_{k} \varrho_{\lambda}^{\prime}\left(x_{k}\right)}{\varrho_{\lambda}\left(x_{k}\right)}\right| \prod_{j} \varrho_{\lambda}\left(x_{j}\right) d^{N} x \\
& \equiv(\max F) \frac{1}{\lambda} E_{\lambda=1}\left[\left|\sum_{j} 1+\frac{x_{j} \varrho^{\prime}\left(x_{j}\right)}{\varrho\left(x_{j}\right)}\right|\right] .
\end{aligned}
$$

We use the Hölder inequality to obtain

$$
\left|\frac{d E_{\lambda}(F)}{d \lambda}\right|^{2} \leqq \frac{(\max F)^{2}}{\lambda^{2}} E_{1}\left(\sum_{j, k}\left[1+\frac{x_{j} \varrho^{\prime}\left(x_{j}\right)}{\varrho\left(x_{j}\right)}\right]\left[1+\frac{x_{k} \varrho^{\prime}\left(x_{k}\right)}{\varrho\left(x_{k}\right)}\right]\right) .
$$

The off-diagonal terms do not contribute because

$$
\left(\int\left[\varrho(x)+x \varrho^{\prime}(x)\right] d x\right)^{2}=\left(\int \frac{d}{d x}(x \varrho(x)) d x\right)^{2}=0,
$$

while the $N$ diagonal terms each contribute

$$
\int\left[1+2 x \varrho^{\prime}(x)+\frac{x^{2} \varrho^{\prime}(x)^{2}}{\varrho(x)}\right] d x \leqq\left(3+M_{\varrho}\right) .
$$

Remark. Recall that the principal purpose of this paper is to show that the quenched correlation length, $\xi$, of disordered Ising ferromagnets obeys a bound of the form $\dot{\xi} \gtrsim\left|\lambda-\lambda_{c}\right|^{-2 / d}$ (where $\gtrsim$ permits modifications of inequalities by constants and logarithms). For future reference, note that by Theorem 2.1 (or Theorem 2.2), it suffices to find a sequence of events $\left(\mathscr{A}_{L}\right)$ such that: (1) the $\left(\mathscr{A}_{L}\right)$ define a critical point $\lambda_{c}$ at which they have the scale invariance property (2.3); and (2) the $\xi_{f}$ defined by $\left(\mathscr{A}_{L}\right)$ satisfies $\infty>\xi \gtrsim \xi_{f}$ for $\lambda<\lambda_{c}$. As we will see, for disordered Ising ferromagnets, it is relatively easy to demonstrate $\xi \gtrsim \xi_{f}$. What is more difficult is to establish the existence of a critical point; for this, we first prove $\xi_{f} \gtrsim \xi$ and then exploit the continuity of finite-volume probabilities.

\section{Disordered Ising Ferromagnetis: Definitions and Preliminaries}

In this section, we define the disordered Ising ferromagnets and derive some basic infinite-volume results, including existence and properties of the quenched correlation length $\xi$. A tool we will use in proving these results is the interacting percolation representation of Ising (and Potts) models introduced by Fortuin and Kasteleyn [11] (hereafter referred to as FK). This representation is briefly reviewed in Sect. 3ii, after which we derive general infinite-volume properties (in Sect. 3iii) and those that pertain specifically to the correlation length (in Sect. 3iv). 
3i. Definition of the Model. Consider the nearest-neighbor disordered Ising ferromagnet with Hamiltonian:

$$
\mathscr{H}=-\sum_{\langle i, j\rangle} J_{i j} \sigma_{i} \sigma_{j},
$$

where the sum is over all nearest-neighbor pairs of $\mathbb{Z}^{d}, \sigma_{i} \in\{-1,+1\}$ are Ising spins and $J_{i j} \geqq 0$ are ferromagnetic couplings. Of course, even for a fixed realization $\left\{J_{i j}\right\}$ of couplings, (3.1) is a formal object. To be precise, we must consider a finite set $\Lambda \subset \mathbb{Z}^{d}$, and set to zero in (3.1) all $J_{i j}$ with both endpoints in $\Lambda^{c} \equiv \mathbb{Z}^{d} \backslash \Lambda$. Let us denote by $\mathbb{B}_{\Lambda}$ the set of all bonds with at least one endpoint in $\Lambda$, and by $\Omega=\Omega(\Lambda)$ $=\left\{J_{i j} \mid\langle i, j\rangle \in \mathbb{B}_{\Lambda}\right\}$ the realization of couplings. Then for each fixed spin configuration $\sigma_{\partial \Lambda}$ on $\partial \Lambda$ ( $=$ the set of points of $\Lambda^{c}$ with a neighbor in $\Lambda$ ), we may define the finite-volume Hamiltonian $\mathscr{H}_{\Lambda}\left(\sigma_{\Lambda} ; \sigma_{\partial \Lambda} ; \Omega\right)$ for any spin configuration $\sigma_{\Lambda} \in\{-1,+1\}^{|\Lambda|}$ in $\Lambda$. The probability measures on $\{-1,+1\}^{|\Lambda|}$ with the weight of any $\sigma_{\Lambda}$ proportional to $\exp \left[-\mathscr{H}_{\Lambda}\left(\sigma_{\Lambda} ; \sigma_{\partial \Lambda} ; \Omega\right)\right]$ are the finite-volume Gibbs measures; from these, the relevant infinite-volume measures may be extracted.

Thus far, we have discussed the Gibbs measures for a fixed realization $\Omega=\left\{J_{i j}\right\}$ of couplings. Henceforth, we will take the $J_{i j}$ to be i.i.d. random variables with distribution $\varrho\left(J_{i j}\right)$ for any given bond. The product measure on $\mathbb{B}_{\Lambda}$ is given by

$$
P\left(D_{\Lambda} \Omega\right) \equiv \prod_{\langle i, j\rangle \in \mathbb{B}_{\Lambda}} \varrho\left(J_{i j}\right) .
$$

We will consider only ferromagnetic distributions, i.e. $\operatorname{Prob}_{\varrho}\left[J_{i j}<0\right]=0$. It turns out that we must put a few additional (mild) conditions on the distribution $\varrho$. These conditions are required to ensure monotonicity of the correlation length, and are discussed explicitly in the Remark preceding Proposition 3.5 in Sect. 3iv.

In the random spin systems, it is natural to consider quenched averages for functions of spin configurations: Let $A\left(\sigma_{\Lambda}\right)$ be any (bounded) function of the spin configurations in $\Lambda$. For fixed boundary conditions, $\sigma_{\partial \Lambda}$, and fixed sample of couplings, $\Omega=\left\{J_{i j} \mid\langle i, j\rangle \in \mathbb{B}_{\Lambda}\right\}$, the thermal expectation of $A$ is simply:

$$
\langle A\rangle_{\Lambda, \sigma_{\partial \Lambda}, \Omega}=Z_{\Lambda, \sigma_{\partial \Lambda}, \Omega} \sum_{\sigma_{\Lambda}} \exp \left[-\mathscr{H}\left(\sigma_{\Lambda} ; \sigma_{\partial \Lambda} ; \Omega\right)\right] A\left(\sigma_{\Lambda}\right),
$$

where $Z_{\Lambda, \sigma_{\partial \Lambda}, \Omega}$ is the appropriate partition function.

The quenched expectation of $A$ is then given by averaging the thermal expectation with respect to the coupling distribution

$$
{\overline{\langle A\rangle_{\Lambda, \sigma_{\partial \Lambda}, \varrho}}}=\int\langle A\rangle_{\Lambda, \sigma_{\partial \Lambda}, \Omega} P\left(D_{\Lambda} \Omega\right) \text {. }
$$

Remarks. (a) For notational convenience, we have absorbed the usual factor of $\beta\left(\equiv 1 / k_{B} T\right)$ into the $J_{i j}$. Thus all temperature dependence will be tracked through the distribution, $\varrho$, of coupling strengths.

(b) For the purposes of expositional clarity, we will omit any explicit reference to the so-called "random site" problem, in which the $J_{i j}$ are given by

$$
J_{i j}=\tau_{i} \tau_{j}
$$

with the $\left\{\tau_{i}\right\}$ a collection of i.i.d. random site variables. The generalization of our results to these problems requires only the substitution of the word "site" for the word "bond" at appropriate points in the derivation. 
3ii. The FK Representation. In [11], Fortuin and Kasteleyn introduced a representation for the Ising (and general $q$-state Potts) spin systems which illustrated the close connection between these problems and independent percolation. Recently, this representation has proved useful in the context of both uniform and disordered ferromagnets $[12,13]$. The reader is referred to [12] for more detailed discussions of what follows.

First we define the bond configurations. Again let $\Lambda \subset \mathbb{Z}^{d}$ be a finite set and denote by $\mathbb{B}_{\Lambda}$ the set of all bonds with at least one endpoint in $\Lambda$. A configuration $\omega \equiv \omega(\Lambda)$ on $\mathbb{B}_{\Lambda}$ consists of a number $\omega_{i j} \in\{0,1\}$ assigned to each $\langle i, j\rangle \in \mathbb{B}_{\Lambda}$. When $\omega_{i j}=1$, the bond is said to be occupied; otherwise it is called vacant. When convenient, $\omega(\Lambda)$ may be viewed as a subset of $\mathbb{B}_{\Lambda}$.

For the pure bond percolation problem, the bonds are assigned independent occupation probabilities $p_{i j} \in[0,1]$, which are usually taken to be uniform (i.e. $p_{i j} \equiv p$ ). Thus the probability of observing $\omega$ is given by

$$
\operatorname{Prob}(\omega)=\prod_{\langle i, j\rangle} p_{i j}^{\omega_{i j}}\left(1-p_{i j}\right)^{\left(1-\omega_{\imath \jmath}\right)}
$$

Fortuin and Kasteleyn discovered that Ising ferromagnets with couplings $\left\{J_{i j} \mid\langle i, j\rangle \in \mathbb{B}_{\Lambda}\right\}$ generate a percolation-like problem with weights

$$
\mathscr{W}(\omega) \propto \prod_{\langle i, j\rangle} p_{i j}^{\omega_{i j}}\left(1-p_{i j}\right)^{\left(1-\omega_{\imath \jmath}\right)} 2^{c(\omega)},
$$

where $c(\omega)$ is the number of connected components of $\omega$ and the $p_{i j}$ are given in terms of the $J_{i j}$ by

$$
p_{i j}=1-e^{-J_{i j}}
$$

The quantity $c(\omega)$ may be defined in a number of distinct ways depending on the boundary conditions. Of relevance here are the free boundary problems, where one simply counts the number of connected components, and "wired" problems, where one identifies all clusters attached to $\partial \Lambda$ as part of the same connected component. These correspond to free and "plus" boundary conditions, respectively, in the original spin system.

When the $p_{i j}$ are constant, (3.7) provides a representation of the uniform spin system. However, there is no difficulty in posing the FK random cluster problems against a background where the $p_{i j}$ are themselves random variables. Thus, in finite volume, we may take a given realization $\Omega$ of the $p_{i j}\left(\right.$ or $\left.J_{i j}\right)$, and construct according to the weights in Eq. (3.7) - the FK free or wired measures. These will be denoted, respectively, by $\mu_{\Lambda, f, \Omega}(-)$ and $\mu_{\Lambda, w, \Omega}(-)$. The quenched expectation of a random cluster event, $\mathscr{A}$, is again defined by averaging over the coupling distribution; as before, these expectations will be denoted by overbars:

$$
\bar{\mu}_{\Lambda, i, \varrho}(\mathscr{A})=\int \mu_{\Lambda, i, \Omega}(\mathscr{A}) P\left(D_{\Lambda} \Omega\right),
$$

where $i=$ free $(f)$ or wired $(w)$.

It turns out that various correlation functions in the spin system correspond to FK probabilities of certain events. Indeed, in [12] it was observed that if $f\left(\sigma_{a}, \ldots, \sigma_{z}\right)$ is any local function, then $\langle f\rangle_{\Lambda_{n}, i, \Omega}(i=$ " + " or free) may be expressed as sums and differences of wired or free FK probabilities of the events that various 
subsets of the sites $\{a, \ldots, z\}$ belong to the same connected component. For example, the spin-spin correlation in finite volume is given by

$$
\left\langle\sigma_{x} \sigma_{y}\right\rangle_{\Lambda, i, \Omega}=\mu_{A, i, \Omega}(x \text { is connected to } y) .
$$

Moreover, it has been shown [7, 12] (following [17]) that, with probability one, the spontaneous magnetization is equal to

$m(\varrho)=\lim _{\Lambda \uparrow \mathbb{Z}^{d}} \frac{1}{|\Lambda|} \sum_{i \in \Lambda}\left\langle\sigma_{i}\right\rangle_{\Lambda,+, \Omega} \equiv \lim _{\Lambda \uparrow \mathbb{Z}^{d}} \frac{1}{|\Lambda|} \sum_{i \in \Lambda} \mu_{\Lambda, w, \Omega}(i$ is connected to $\partial \Lambda)$.

3iii. FKG Properties and Infinite-Volume Limits. In finite volumes, with specified boundary conditions, the quenched measures $\bar{\mu}_{A, i, \varrho}(-)$ and $\overline{\langle-\rangle}_{A, i, e}$ are welldefined objects. Here we review the notion of FKG monotonicity [14], and use this monotonicity to establish the existence of infinite-volume limits.

Definition. (FKG Ordering, Measures and Domination): If $w_{1}$ and $w_{2}$ are "configurations" which assign a number $w_{1}(\langle i, j\rangle), w_{2}(\langle i, j\rangle)$ to each bond of the lattice, we say that

$$
w_{1}>w_{2}
$$

if for each $\langle i, j\rangle, w_{1}(\langle i, j\rangle) \geqq w_{2}(\langle i, j\rangle)$. This defines a partial order on the space of bond configurations. A function which is non-decreasing with respect to this partial order is said to be positive. An event is said to be positive if its indicator function is positive. A measure $v$ is said to be an FKG measure if for every pair of positive functions, $f$ and $g$ :

$$
v(f g) \geqq v(f) v(g) .
$$

If $v_{1}$ and $v_{2}$ are two measures, we say that $v_{1}$ FKG dominates $v_{2}$, denoted by

$$
v_{1} \underset{\mathrm{FKG}}{\gtrless} v_{2},
$$

if for every positive function $g$ :

$$
v_{1}(g) \geqq v_{2}(g) .
$$

It turns out that both simple product (e.g. percolation) and the interacting FK random cluster measures are FKG.

Lemma 3.1a [15]. Bernoulli measures are FKG.

Lemma 3.1b $[11,12,14,16]$. For any fixed realization, $\Omega$, the Ising random cluster measures in finite volume with free or wired boundary conditions are FKG. Furthermore, for finite sets $\Lambda_{1}, \Lambda_{2} \subset \mathbb{Z}^{d}$ with $\Lambda_{1} \supset \Lambda_{2}$, the free boundary random cluster measures, $\mu_{\Lambda_{1}, f, \Omega}(-)$ and $\mu_{\Lambda_{2}, f, \Omega}(-)$ satisfy

$$
\mu_{\Lambda_{1}, f, \Omega}(-) \underset{\mathrm{FKG}}{\geqq} \mu_{\Lambda_{2}, f, \Omega}(-),
$$

while the wired random cluster measures, $\mu_{\Lambda_{1}, w, \Omega}(-)$ and $\mu_{\Lambda_{2}, w, \Omega}(-)$ satisfy the opposite inequality

$$
\mu_{\Lambda_{2}, w, \Omega}(-) \underset{\mathrm{FKG}}{\gtrless} \mu_{\Lambda_{1}, w, \Omega}(-) .
$$


With the above in mind, one can easily establish the existence of various infinite-volume measures:

Theorem 3.2a $[11,12,14,16]$. For any fixed realization, $\Omega$, infinite-volume limits exist for the free and wired boundary Ising random cluster measures, and for the free and plus (or minus) boundary Ising spin measures.

Theorem 3.2b. For the four systems specified above, infinite-volume limits exist for the quenched measures. Furthermore, infinite-volume limits and disorder averages may be commuted.

Proof. The existence of quenched measures follows easily from monotonicity (in the volume) of the quenched probabilities of positive cylinder events. The fact that one may commute infinite-volume limits and disorder averages is a consequence of the monotone convergence theorem.

Before turning to the correlation length in the high-temperature phase, let us show that we can ignore boundary conditions in that phase. As usual, the hightemperature phase is defined by the vanishing of the spontaneous magnetization (3.11). The statement we need is essentially contained in [12], where it is shown that whenever the magnetization vanishes, the limiting Gibbs state (or corresponding infinite-volume FK state) is unique. Noting that $m(\varrho)$ can also be obtained via quenching (as shown e.g. in $[7,12,13]$ ):

$$
m(\varrho)={\overline{\left\langle\sigma_{0}\right\rangle_{+, \varrho}}}=\bar{\mu}_{w, \varrho}(0 \text { belongs to an infinite cluster }),
$$

and that infinite-volume limits and quenching commute, it follows that:

Theorem 3.3. For random Ising ferromagnets with independent disorder, and the associated random cluster models, whenever $m(\varrho)=0$, with probability one, the limiting infinite-volume state is unique. Furthermore, $m(\varrho)=0$ also implies uniqueness of the quenched infinite-volume measure.

3iv. Existence and Properties of the Correlation Length. With the results of the previous subsection in mind, the existence and general properties of the quenched correlation length are readily established.

Proposition 3.4. Let $\overline{\langle-\rangle_{e}}$ denote the (unique) infinite-volume quenched spin measure in the single-phase regime. Then the limit

$$
-\frac{1}{\xi(\varrho)} \equiv \lim _{x \rightarrow \infty} \frac{1}{|x|} \log \overline{\left\langle\sigma_{0} \sigma_{x}\right\rangle_{\varrho}}
$$

exists for any sequence of points along a coordinate axis. Furthermore,

$$
\overline{\left\langle\sigma_{0} \sigma_{x}\right\rangle_{\varrho}} \leqq e^{-|x| / \xi}
$$

holds uniformly in $x$; or, in general,

$$
\overline{\left\langle\sigma_{0} \sigma_{x}\right\rangle_{\varrho}} \leqq e^{-\|x\| / \xi},
$$

where $\|x\| \equiv \max \left\{\left|x_{1}\right|, \ldots,\left|x_{d}\right|\right\}$. 
Proof. Let $x, y \in \mathbb{Z}^{d}$. Then, for each configuration $\Omega$,

$$
\left\langle\sigma_{0} \sigma_{x}\right\rangle_{\Omega} \geqq\left\langle\sigma_{0} \sigma_{y}\right\rangle_{\Omega}\left\langle\sigma_{y} \sigma_{x}\right\rangle_{\Omega}
$$

this is either the $2^{\text {nd }}$ Griffiths inequality or the FKG inequality in the FK representation. For either reason, both $\left\langle\sigma_{0} \sigma_{x}\right\rangle$ and $\left\langle\sigma_{y} \sigma_{x}\right\rangle$ are $P$-increasing functions; hence, by the FKG inequality,

$$
\overline{\left\langle\sigma_{0} \sigma_{x}\right\rangle_{\varrho}} \geqq \overline{\left\langle\sigma_{0} \sigma_{y}\right\rangle_{\varrho}} \overline{\left\langle\sigma_{y} \sigma_{x}\right\rangle_{\varrho}} \text {. }
$$

The desired results now follow from standard subadditive arguments (see e.g. [18]).

Remark. In order to ensure that a critical point exists, we will need monotonicity and continuity properties of the correlation length. As shown below, these in turn are guaranteed by FKG domination of measures as a function of the parameter by which we approach the transition. If $\varrho_{1}$ and $\varrho_{2}$ are single-bond measures for the spin system, it turns out that the necessary and sufficient condition for the associated product measures to satisfy $P_{1} \underset{\text { FKG }}{\gtrless} P_{2}$ is that the inequality

$$
\operatorname{Prob}_{\varrho_{1}}(J>x) \geqq \operatorname{Prob}_{\varrho_{2}}(J>x)
$$

hold for all $x$. Thus if the $J_{i j}$ are given in terms of "physical" couplings $\mathscr{J}_{i j}$ and a temperature parameter $\beta$ :

$$
J_{i j}=\beta \mathscr{J}_{i j}
$$

then increasing $\beta$ (holding the distribution of the $\mathscr{J}_{i j}$ fixed) increases, in the sense of FKG, the coupling distribution. Now suppose that we intend to approach the transition by varying the mixing (disorder) parameter of a convex combination of two (single-bond) measures. Then, provided that one single-bond measure FKG dominates the other, we obtain FKG domination of the product measures as a function of disorder. Thus, in the latter case, we will require that the two singlebond measures in the convex combination have an FKG dominance relation.

Proposition 3.5. Let $\left(\varrho_{\alpha} \mid \alpha \in \mathbb{R}\right)$ be a one-parameter family of measures. Suppose that $\left(\varrho_{\alpha}\right)$ is continuous in the sense that for each interval $[a, b] \subset[0,1]$

$$
\lim _{\alpha \rightarrow \alpha_{0}} \operatorname{Prob}_{\alpha}(p \in[a, b])=\operatorname{Prob}_{\alpha_{0}}(p \in[a, b]) .
$$

Further suppose that whenever $\alpha>\alpha^{\prime}$

$$
\varrho_{\alpha} \underset{\mathrm{FKG}}{\gtrless} \varrho_{\alpha^{\prime}} \cdot
$$

Then $\xi^{-1}(\alpha)$ is a monotone non-increasing, left-continuous function.

Proof. Monotonicity is obvious from the FKG inequalities. Continuity follows from a standard one-dimensional construction (i.e. calculating $\xi$ in $L^{d-1} \times \mathbb{Z}$ strips). Exploiting monotonicity in the volume (of the type described in Lemma $3.1 \mathrm{~b}$ ) and continuity of finite-volume probabilities (which follows from the above continuity hypothesis), this construction expresses $\xi^{-1}$ as the decreasing limit of continuous decreasing functions, which is therefore left-continuous. For more details, see e.g. [18]. 


\section{Correlation Length Bounds for Disordered Ising Ferromagnets}

4i. Construction of the Finite-Size Scaling Event. At low temperatures, when there is positive spontaneous magnetization in the infinite-volume system, finite volumes will align according to the spin configuration at the boundary. On the other hand, in the high-temperature phase, the statistical behavior of large blocks should be relatively insensitive to boundary conditions. We will consider cubes

$$
C_{L}=\left\{x \in \mathbb{Z}^{d} \mid\|x\| \leqq L\right\},
$$

and, for fixed $\Omega$, study the finite-volume Gibbs measures $\langle-\rangle_{C_{L},+, \Omega}$. At sufficiently low temperature, one would expect that, with probability close to one, the inner portion of the cubes will magnetize. Thus we define the magnetic response (of the inner portion of the cube $C_{3 L}$ ):

$$
M_{L}(\Omega) \equiv\left\langle\sum_{i \in C_{L}} \sigma_{i}\right\rangle_{C_{3 L},+, \Omega}
$$

It is in fact easy to show that when $m(\varrho)>0, M_{L}(\Omega)$ is typically of the order of $L^{d}$. On the other hand, when the correlation length is finite, one envisions that $M_{L}(\Omega)$ will tend to zero exponentially fast in $L{ }^{2}$ It is therefore natural to define the finitesize scaling event

$$
\mathscr{A}_{L}=\left\{\Omega \mid M_{L}(\Omega) \geqq a_{1}\right\},
$$

and hence

$$
\xi_{f}=\max \left\{L \mid P\left(\mathscr{A}_{L}\right)>a_{2}\right\}
$$

with $a_{1}$ and $a_{2}$ constants of order unity. Recall that $P$ denotes the probability with respect to the product measure (3.2).

4ii. Equivalence of Correlation Lengths. The principal result of this section will be to demonstrate that $\xi_{f} \approx \xi$. Explicitly, we will show that

$$
\frac{\xi_{f}(\varrho)}{c_{1} \log \xi_{f}(\varrho)+c_{2}} \leqq \xi(\varrho) \leqq c_{3} \xi_{f}(\varrho)+c_{4}
$$

with the $c_{i}$ uniform. Our particular choice of constants turns out to be

$$
\begin{gathered}
a_{1}=a_{2} \equiv a=\frac{1}{2}\left[e^{-1}\right]\left[5^{-d}\right]\left[6^{-(d-1)}\right], \quad c_{1}=2 d-1, \\
c_{2}=\log \left[\frac{d(12)^{d}}{3 a^{2}}\right], \quad c_{3}=c_{4}=6 .
\end{gathered}
$$

This, however, is a function of the details of our proof. The actual critical scaling of $M_{L}(\Omega)$ undoubtedly involves powers of $L$; our inability to characterize precisely the critical behavior is, to some extent, reflected in the $\log \xi_{f}$ on the left-hand side of Eq. (4.5). It is worth noting that upper and lower bounds of the form (4.5) without

\footnotetext{
${ }^{2}$ If the transition is first order (which we will later rule out for the Ising case), at the point where the high- and low-temperature phases coexist, $M_{L}$ will scale like $L^{d}$, despite the fact that $\xi$ is finite. Presumably, this happens in the $q$-state Potts models in high enough dimension when $q>2$
} 
the modification by $\log s$ have recently been obtained for the case of twodimensional percolation by Kesten [19].

Remark. At high enough temperature, the condition $P\left(\mathscr{A}_{L}\right)>a$ will fail for all $L$. In this case, we will define $\xi_{f}$ (and $\xi_{f} / \log \xi_{f}$ ) to be zero. Hence, if $\xi \ll 1$, one should not take the equivalence of $\xi$ and $\xi_{f}$ too seriously. Fortunately, in these regimes, expansion techniques provide quite efficient algorithms for computing $\xi$ (see [20]).

The easy half of the equivalence is a consequence of the following.

Proposition 4.1. $P\left(M_{L}\right) \leqq[$ Const $] L^{[2 d-1]} e^{-L / \xi}$ with Const (unimportantly) equal to $\frac{1}{3} d[12]^{d}$.

Proof. Suppose that for some finite $\Lambda \subset \mathbb{Z}^{d}$ and fixed realization, $\Omega$, we wish to compute $\left\langle\sigma_{i}\right\rangle_{\Lambda,+, \Omega}$ for $i \in \Lambda$. Let us define $\bar{\Lambda}$ by identifying all $j \in \partial \Lambda$ as a single site. On this site, we place an Ising spin, $\mathbb{S}$, which couples to all the neighbors $k \in \Lambda$ of the $j \in \partial \Lambda$ with strength $J_{k j}$. For a small $d=2$ lattice, this is illustrated in Fig. 1. Denoting by $\langle-\rangle_{\Lambda, \Omega}$ the measures in these "wired" lattices, it is not hard to see that

$$
\left\langle\sigma_{i}\right\rangle_{\Lambda,+, \Omega}=\left\langle\sigma_{i} \mathbf{S}\right\rangle_{\Lambda, \Omega} .
$$

Denote by $\partial_{I} \Lambda$ the internal boundary of $\Lambda$ (i.e. the sites in $\Lambda$ with neighbors in $\partial \Lambda$ ). Then, by the Lieb-Simon inequality $[21,22]$,

$$
\left\langle\sigma_{i} \mathbf{S}\right\rangle_{\Lambda, \Omega} \leqq \sum_{k \in \partial_{I} \Lambda}\left\langle\sigma_{i} \sigma_{k}\right\rangle_{\Lambda, f, \Omega}\left\langle\sigma_{k} \mathbf{S}\right\rangle_{\Lambda, \Omega} \leqq \sum_{k \in \partial_{I} \Lambda}\left\langle\sigma_{i} \sigma_{k}\right\rangle_{\Lambda, f, \Omega}
$$

Note that in the final expression, expectations are evaluated with free boundary conditions. However, for any $\Omega,\left\langle\sigma_{i} \sigma_{k}\right\rangle_{\Lambda, f, \Omega}$ does not exceed the corresponding average in the infinite-volume limit. Thus, by Proposition 3.4, in the single-phase regime

$$
\overline{\left\langle\sigma_{i}\right\rangle_{\Lambda}++} \leqq \sum_{k \in \partial_{I} \Lambda} e^{-\|k-i\| / \xi}
$$

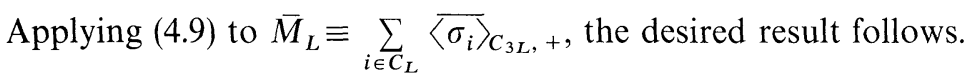

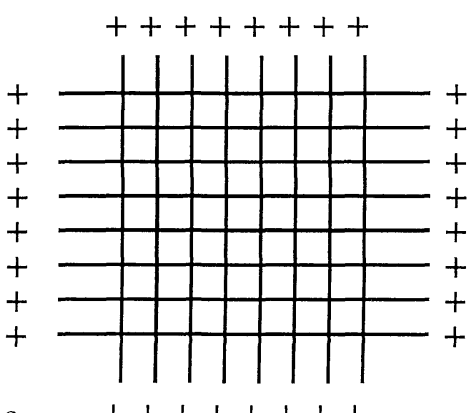

a

$++++++$

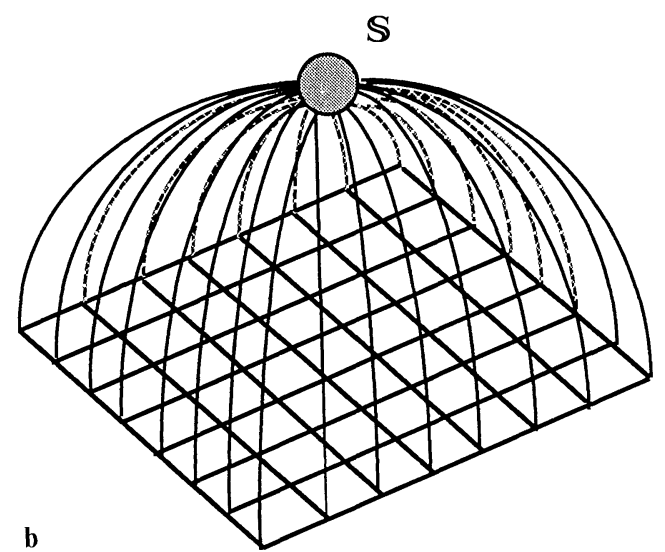

Fig. 1a and b. A wired lattice (a) and its equivalent representation (b) 
Corollary. There are constants $c_{1}$ and $c_{2}$ such that the correlation length $\xi_{f}$ satisfies

$$
\xi \geqq \frac{\xi_{f}}{c_{1} \log \xi_{f}+c_{2}}
$$

in the single-phase regime.

Proof. If $\xi_{f}$ is zero or $\xi$ is infinite, there is nothing to prove. Otherwise, at the scale $\xi_{f}$, by definition:

$$
P\left(M_{\xi_{f}} \geqq a\right)>a,
$$

so that

$$
\bar{M}_{\xi_{f}}>a^{2}
$$

Thus

$$
[\text { Const }] \xi_{f}^{[2 d-1]} e^{-\xi_{f} / \xi} \geqq a^{2},
$$

and the result follows easily with the constants $c_{1}$ and $c_{2}$ as given in Eq. (4.6).

Remark. Although straightforward, the preceding relied on the Lieb-Simon inequality [21, 22], which does not hold for general spin systems. We require both the lower bound on $\xi$ and the upper bound proved below to establish, for these systems, the continuous divergence of $\xi$, and hence the existence of a critical point for the $\left(\mathscr{A}_{L}\right)$. Although somewhat more difficult, Theorem 4.2 can, in fact, be established e.g. for the $q$-state Potts models and, perhaps, in even greater generality. Such results are, however, worthless without an analogue of Proposition 4.1 and its corollary.

We now attend to the major task of this section.

Theorem 4.2. The correlation length $\xi_{f}$ satisfies

$$
\xi \leqq 6 \xi_{f}+6
$$

in the single phase regime.

Remark. At the core of our proof is the fact that the quenched expectation of a certain event obeys a rescaling inequality of the sort introduced in [23]. Unfortunately, this event, which is defined naturally in the FK representation, does not admit a particularly transparent formulation in the spin language. We will thus define the event below, and state the rescaling lemma - relegating the proof of the lemma to the appendix. Assuming the lemma, we may proceed with a relatively succinct proof of Theorem 4.2.

Let $\Omega$ denote a fixed coupling configuration and consider the regions

$$
B_{L}=\left\{x \in \mathbb{Z}^{d}|| x_{1}|, \ldots,| x_{d-1}|\leqq 12 L ;| x_{d} \mid \leqq 3 L\right\} .
$$

We will be interested in the FK wired measures on $B_{L}, \mu_{B_{L}, w, \Omega}(-)$. Although the wiring will occur on $B_{L}$, the events that will concern us will take place in the inner box $\left\{\left|x_{1}\right|, \ldots,\left|x_{d-1}\right| \leqq 6 L ;\left|x_{d}\right| \leqq 3 L\right\}$. If $\omega$ is a random cluster configuration in $B_{L}$, we will say that the event $\mathscr{N}_{L}$ occurs if there is no crossing by the occupied bonds of $\omega$, which lies entirely within the inner box, and which extends from any of the sites on 


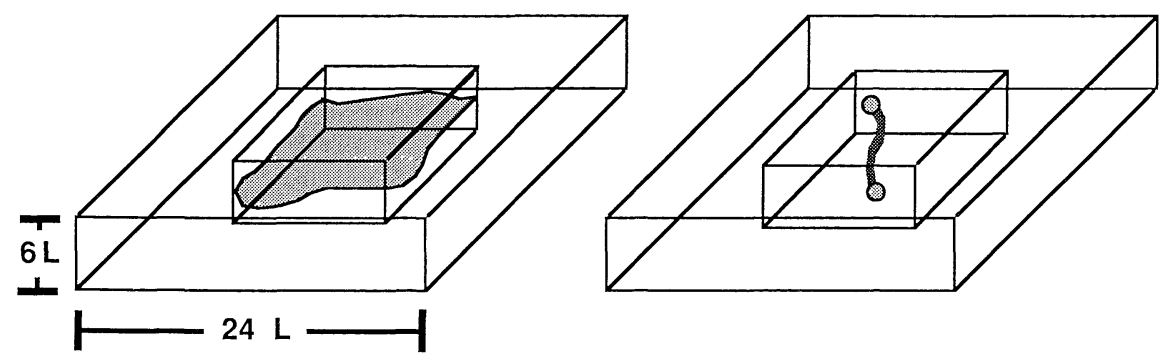

Fig. 2. The rescaling event and its complement

the "bottom face": $\left\{\left|x_{1}\right|, \ldots,\left|x_{d-1}\right| \leqq 6 L ;\left|x_{d}\right|=-(3 L-1)\right\}$ to any of the sites on the "top face": $\left\{\left|x_{1}\right|, \ldots,\left|x_{d-1}\right| \leqq 6 L ;\left|x_{d}\right|=+(3 L-1)\right\}$. The use of FK bonds is obviously a better choice for describing the complementary event $\mathscr{N}_{L}^{c}$. To more concisely describe $\mathscr{N}_{L}$, we use the well-known duality: for each vacant bond, we will occupy its corresponding $(d-1)$-cell on the dual lattice. If we endow these dual cells with the standard connective properties, i.e. two $(d-1)$-cells are connected if they have a common $(d-2)$-cell in their boundaries, the event $\mathscr{N}_{L}$ may be defined as follows:

$$
\begin{aligned}
& \mathscr{N}_{L}=\{\omega \mid \text { there is a sheet of dual }(d-1) \text {-cells in the region } \\
&\left.\left\{\left|x_{1}\right|, \ldots,\left|x_{d-1}\right| \leqq\left(6 L+\frac{1}{2}\right) ;\left|x_{d}\right| \leqq 3 L-\frac{1}{2}\right)\right\} \\
&\text { which separates } \left.x_{d} \leqq-3 L \text { from } x_{d} \geqq+3 L\right\} .
\end{aligned}
$$

The events $\mathscr{N}_{L}$ and $\mathscr{N}_{L}^{c}$ are illustrated, for $d=3$, in Fig. 2.

The rescaling lemma may be stated as follows:

Lemma 4.3. Let $\alpha<1$, and suppose that for some $L$

$$
\bar{\mu}_{B_{L}, w, \varrho}\left(\mathscr{N}_{L}\right) \geqq 1-\alpha 5^{-d} .
$$

Then at scale $2 L$

$$
\bar{\mu}_{B_{2 L}, w, \varrho}\left(\mathscr{N}_{2 L}\right) \geqq 1-\alpha^{2} 5^{-d}
$$

Proof. See appendix.

From this, it is not difficult to prove the equivalence of $\xi_{f}$ and $\xi$.

Proof of Theorem 4.2. We assume that $\xi_{f}<\infty$ (otherwise we are done), and denote by $L^{*}$ any length larger than $\xi_{f}$ (e.g. $\left.L^{*}=\xi_{f}+1\right)$. At scale $L^{*}$, we have, by definition,

$$
P\left(\mathscr{A}_{L^{*}}^{c}\right) \geqq 1-a .
$$

We claim that this implies $\bar{\mu}_{B_{L^{*}, w, \varrho}}\left(\mathscr{N}_{L}\right) \geqq 1-e^{-1} 5^{-d}$.

Indeed, let $\Omega$ be a coupling configuration and consider $\mu_{C_{3 L^{*}, w}, \Omega}(-)$, the wired FK measures on the cubes $C_{3 L^{*}}$. Let us denote by $\mathscr{S}_{L}$ the event $\mathscr{S}_{L}=\left\{\omega \mid\right.$ there is no crossing between $C_{L}$ and $\partial C_{3 L}$ by occupied bonds $\}$, 

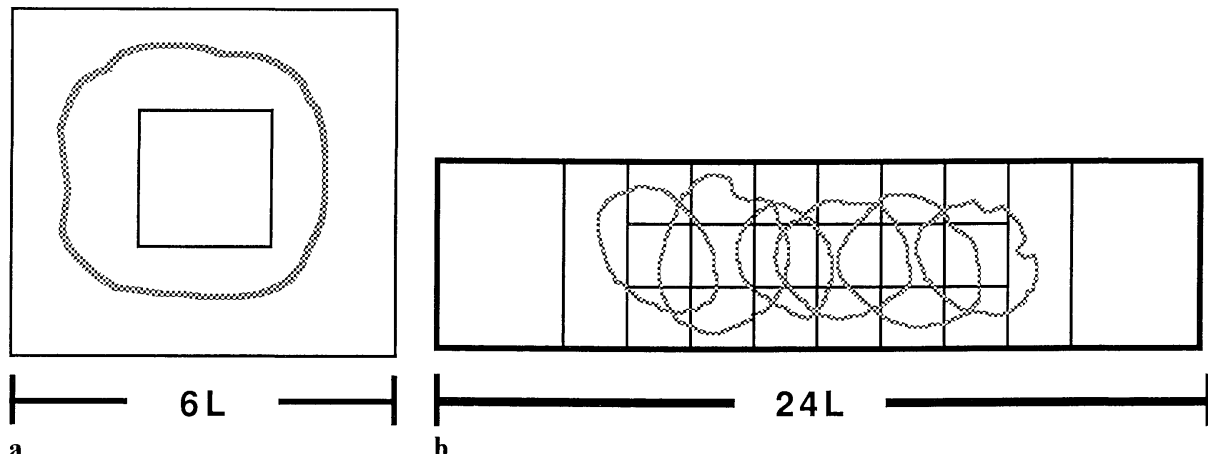
b

Fig. 3. a The event $\mathscr{S}_{L}$. b Translates of $\mathscr{S}_{L}$

or dually

$\mathscr{S}_{L}=\left\{\omega \mid\right.$ there is a surface inside $C_{3 L}$ which separates $C_{L}$ from $\left.\partial C_{3 L}\right\}$ (see Fig. 3a).

By subadditivity, it is seen that

$$
\begin{aligned}
1-\mu_{C_{3 L^{*}, w, \Omega}}\left(\mathscr{S}_{L^{*}}\right) & \leqq \sum_{i \in C_{L^{*}}} \mu_{C_{3 L^{*}, w}, \Omega}\left(i \text { is connected to } \partial C_{3 L}\right) \\
& =\sum_{i \in C_{L^{*}}}\left\langle\sigma_{i}\right\rangle_{C_{3 L^{*},+, \Omega}} \equiv M_{L^{*}}(\Omega) .
\end{aligned}
$$

At scales $L^{*}, \Omega \in \mathscr{A}_{L^{*}}^{c}$ with probability exceeding $1-a$. When this happens we have, by definition, $M_{L^{*}}(\Omega) \leqq a$. Hence,

$$
\bar{\mu}_{C_{3 L^{*}, w},{ }}\left(\mathscr{S}_{L^{*}}\right) \geqq(1-a)^{2} \geqq 1-2 a .
$$

Next, for $x \in \mathbb{Z}^{d}$ consider $\mathrm{T}(x) \mathscr{S}_{L^{*}}$, the event $\mathscr{S}_{L^{*}}$ translated by $x$. As explicitly illustrated in Fig. $3 \mathrm{~b}$ for the two-dimensional case, it is not hard to see that

$$
\mathscr{N}_{L^{*}} \subset \bigcap_{\substack{k_{1}=1 \\ k_{2}=1 \\ \vdots \\ k_{d-1}=1}}^{6} \mathrm{~T}\left(k_{1} L^{*}, \ldots, k_{d-1} L^{*}, 0\right) \mathscr{S}_{L^{*}} \equiv \bigcap_{\vec{k}} \mathrm{~T}\left(\vec{k} L^{*}\right) \mathscr{S}_{L^{*}}
$$

Indeed, the right-hand side of (4.19) implies there is no connection from $\bigcup_{\vec{k}} \mathrm{~T}\left(\vec{k} L^{*}\right) C_{L^{*}}$ to $\bigcup_{\vec{k}} \mathrm{~T}\left(\vec{k} L^{*}\right) \partial C_{3 L^{*}}$ [where we use $\mathrm{T}(x) \Lambda$ to denotes the translation of the set $\Lambda$ by $x]$. We therefore have, by subadditivity,

$$
\mu_{B_{L^{*}, w}, \Omega}\left(\mathscr{N}_{L^{*}}\right) \geqq 1-\sum_{\vec{k}} \mu_{B_{L^{*}, w, \Omega}}\left(\mathrm{T}\left(\vec{k} L^{*}\right) \mathscr{S}_{L^{*}}^{c}\right) .
$$

However, since $C_{3 L^{*}} \subset B_{L^{*}}$, by the domination Lemma $3.1 \mathrm{~b}$, for all the $\vec{k}$,

$$
\mu_{\mathrm{T}\left(C_{\left.3 L^{*}\right), w, \Omega}\right.}(-) \underset{\mathrm{FKG}}{\geqq} \mu_{B_{L^{*}, w, \Omega}}(-) .
$$


Thus, noting that $\mathscr{S}_{L^{*}}^{c}$ is a positive event (i.e. an event for which the indicator function is FKG positive),

$$
\mu_{B_{L^{*}, w, \Omega}}\left(\mathscr{N}_{L^{*}}\right) \geqq 1-\sum_{k} \mu_{\mathrm{T}\left(C_{\left.3 L^{*}\right), w, \Omega}\right.}\left(\mathrm{T}\left(\vec{k} L^{*}\right) \mathscr{S}_{L^{*}}^{c}\right) .
$$

After averaging over disorder, using (4.18) and the definition of $a$ in (4.6), we obtain

$$
\bar{\mu}_{B_{L^{*}, w, \varrho}}\left(\mathscr{N}_{L^{*}}\right) \geqq 1-6^{(d-1)} 2 a=1-e^{-1} 5^{-d}
$$

as claimed.

Iterating the rescaling inequality $n$ times, we have for $L_{n} \equiv 2^{n} L^{*}$,

$$
\bar{\mu}_{B_{L_{n}}, w, \varrho}\left(\mathscr{N}_{L_{n}}\right) \geqq 1-5^{-d} e^{-L_{n} / L^{*}} .
$$

Pasting together $2 d$ translations and reflections of the event $\mathscr{N}_{L}$, as illustrated in Fig. 4 , it is seen that those configurations in which all of these $\mathscr{N}_{L}$ 's occur contribute nothing to the expectation of $\sigma_{0} \sigma_{x}$ if $\|x\| \geqq 6 L$ (cf. Eq. (3.10)). Thus, for any $x$ with $\|x\|=6 L_{n}$, we have

$$
\overline{\left\langle\sigma_{0} \sigma_{x}\right\rangle_{\varrho}} \leqq e^{-\|x\| / 6 L^{*}}
$$

Since, according to Proposition 3.4, the limit $\xi$ exists, Eq. (4.25) implies that $\xi \leqq 6 L^{*}$, provided $L^{*}>\xi_{f}$. Evidently

$$
\xi \leqq 6 \xi_{f}+6 .
$$

4iii. A Critical Point and the Bound $v \geqq 2 / d$. It only remains to prove the existence of a critical point.

Theorem 4.4. Let $\varrho_{\alpha}$ be a continuous, FKG-increasing family of single-bond measures of the type described in Proposition 3.5. Suppose, further, that for $\alpha$ sufficiently small, $\xi(\alpha)<\infty$, while for some $\alpha \in \mathbb{R}, \xi(\alpha)$ is infinite and/or there are multiple Gibbs states. Then there is a critical point, $\alpha_{c}$, at which $\xi$ diverges continuously.

Fig. 4. Decay of correlations

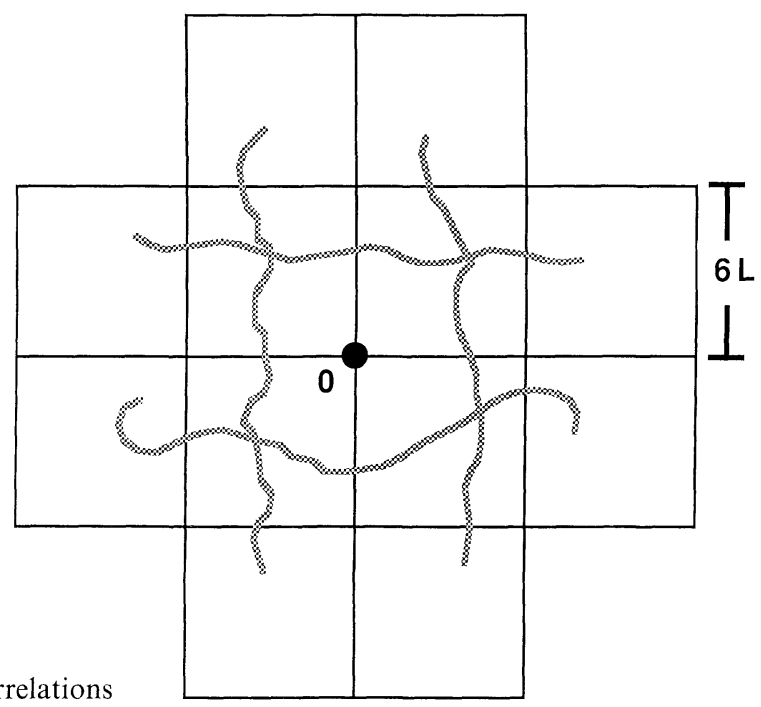


Proof. Let us define $\alpha_{c}$ via

$$
\alpha_{c}=\sup _{\alpha}\{\xi(\alpha)<\infty\} .
$$

Observe that, by hypothesis, $\alpha_{c}$ is nontrivial. By the semicontinuity result of Proposition 3.5,

$$
\xi^{-1}\left(\alpha_{c}\right)=\lim _{\alpha \uparrow \alpha_{c}} \xi^{-1}(\alpha) .
$$

The only danger is that at $\alpha_{c}, \xi$ is finite. Were this the case, by the corollary to Proposition 4.1, we would have $\xi_{f}\left(\alpha_{c}\right)$ finite. Using the continuity of finite volume probabilities, it is seen that, if $\varepsilon$ is sufficiently small, within some scale larger than $\xi_{f}$ (e.g. at scale $\xi_{f}+1$ ), the defining condition in Eq. (4.4) is violated at $\alpha_{c}+\varepsilon$. However, we could then use the argument of Theorem 4.2 to demonstrate that $\xi\left(\alpha_{c}+\varepsilon\right)$ is finite, which contradicts the definition of $\alpha_{c}$.

Evidently, $\xi^{-1}\left(\alpha_{c}\right)=0$ and, in particular, at $\alpha_{c}$ the condition in Eq. (4.4) is satisfied at all scales.

Corollary. Let $\varrho_{\beta}$ or $\varrho_{\lambda}$ be a family of measures of the type described in the Remark preceding Proposition 3.5, i.e. $\varrho_{\beta}$ given by

$$
p_{i j}={ }_{d} 1-\exp \left[-\beta \mathscr{J}_{i j}\right]
$$

with the distribution of physical couplings $\mathscr{J}_{i j}$ fixed (and sufficiently smooth to satisfy condition $(2.7 \mathrm{M})$ ), or $\varrho_{\lambda}$ given by

$$
p_{i j}={ }_{d}\left\{\begin{array}{ll}
p_{i j}^{(1)} ; & \text { with prob. } \lambda \\
p_{i j}^{(2)} ; & \text { with prob. }(1-\lambda)
\end{array},\right.
$$

where $p_{i j}^{(1)}$ and $p_{i j}^{(2)}$ are distributed according to probability measures $\varrho_{1}, \varrho_{2}$ satisfying

$$
\varrho_{1} \underset{\text { FKG }}{\gtrless} \varrho_{2} \text {. }
$$

Then under any conditions which ensure a nontrivial $\beta_{c}$ or $\gamma_{c}$, $\xi$ is bounded below, up to logarithmic corrections, by $\varepsilon^{-2 / d}$, where $\varepsilon=\left|\beta_{c}-\beta\right|$ or $\left|\lambda_{c}-\lambda\right|$ respectively.

Proof. Let us quickly define $\tilde{\xi}_{f}$ by the condition

$$
\tilde{\xi}_{f}=\max \left\{L \mid P\left(M_{L} \geqq a\right)>\frac{1}{2} a\right\} .
$$

By checking the arguments of Proposition 4.1 and Theorem 4.2, it is easily seen that

$$
\xi_{f} \approx \tilde{\xi}_{f} \approx \xi
$$

Applying Theorem 2.1 for the convex case or (the multiplicative version of) Theorem 2.2 for the scaling case, we have, at a distance $\varepsilon$ from the critical point, $\widetilde{\xi}_{f}$ $\geqq K \varepsilon^{-2 / d}$ with $K$ a constant, or

$$
\xi(\varepsilon) \geqq K^{\prime}|\log \varepsilon| \varepsilon^{-2 / d}
$$

for $\varepsilon$ small enough. 


\section{Appendix. The Rescaling Lemma}

Here we prove Lemma 4.3, which is a rescaling inequality along the lines of the percolation inequalities of $[23,7]$ (see also [18]) for the event $\mathscr{N}_{L}$. [See Eqs. (4.13)-(4.14) for relevant definitions.] The only essential difference between the proof below and the percolation arguments is that here some attention must be paid to boundary conditions. To ease the exposition, we will run through the proof in two dimensions; afterwards, we will indicate all modifications necessary for the higher-dimensional cases.

Proof of Lemma 4.3. We start by restricting to $d=2$. Ultimately, we will be concerned with wired boundary conditions on the region $B_{2 L}$. There, the event $\mathscr{N}_{2 L}$ is produced with the stated probability estimate by showing that, with not unreasonable probability, $\mathscr{N}_{2 L}$ occurs either in the upper half or in the lower half of $B_{2 L}$ (denoted by $B_{2 L}^{t}$ and $B_{2 L}^{b}$ respectively) with wired boundary conditions imposed on these smaller regions. Explicitly, let us denote by $\mathscr{N}_{2 L}^{t}\left(\mathscr{N}_{2 L}^{b}\right)$ the event that $\mathscr{N}_{2 L}$ occurs entirely in the region $x_{2}>0\left(x_{2}<0\right)$. Shortly, we will establish the quenched estimate

$$
\bar{\mu}_{B_{2 L}^{t}, w, \varrho}\left(\mathscr{N}_{2 L}^{t}\right) \geqq\left[\bar{\mu}_{B_{L}, w, \varrho}\left(\mathscr{N}_{L}\right)\right]^{5},
$$

so that if $\bar{\mu}_{B_{L}, w, \varrho}\left(\mathscr{N}_{L}\right) \geqq 1-\frac{1}{25} \alpha$, then $\bar{\mu}_{B_{2 L}^{t}, w, \varrho}\left(\mathscr{N}_{2 L}^{t}\right) \geqq 1-\frac{1}{5} \alpha$. From this, the desired bound is straightforward. Indeed,

$$
1-\mu_{B_{2 L}, w, \Omega}\left(\mathscr{N}_{2 L}\right) \leqq \mu_{B_{2 L}, w, \Omega}\left(\left[\mathcal{N}_{2 L}^{t}\right]^{c} \cup\left[\mathscr{N}_{2 L}^{b}\right]^{c}\right)
$$

(where $[-]^{c}$ denotes complementation), since the probability that $\mathscr{N}_{2 L}$ does not happen does not exceed the probability that it fails only in the top and bottom halves of $B_{2 L}$. The insertion of a wiring configuration along the line $x_{2}=0$ only enhances the right-hand side of (A.2) because $\left[\mathscr{N}_{2 L}^{t}\right]^{c},\left[\mathcal{N}_{2 L}^{b}\right]^{c}$ and an additional wire are all FKG-positive events. However, the wire thermally decouples the two events and we get, as a further degradation of (A.2),

$$
1-\mu_{B_{2 L}, w, \Omega}\left(\mathscr{N}_{2 L}\right) \leqq\left[\mu_{B_{2 L}^{t}, w, \Omega}\left(\left[\mathscr{N}_{2 L}^{t}\right]^{c}\right)\left[\mu_{B_{2 L}^{b}, w, \Omega}\left[\mathscr{N}_{2 L}^{b}\right]^{c}\right]\right.
$$

Ostensibly, the right-hand side is still correlated (the wrong way) for a disorder average. However, the first factor depends only on $\Omega$ in the region $B_{2 L}^{t}$ and the second on $\Omega$ restricted to $B_{2 L}^{b}$. Thus, the final averaging may be performed in the separate regions which, using the pruported bound

$$
\bar{\mu}_{B_{2 L}^{t}, w}\left(\left[\mathscr{N}_{2 L}^{t}\right]^{c}\right) \leqq \frac{1}{5} \alpha,
$$

allows

$$
1-\bar{\mu}_{B_{2 L}, w, \Omega}\left(\mathscr{N}_{2 L}\right) \leqq \frac{1}{25} \alpha^{2} .
$$

This is the stated estimate for $d=2$.

We now face up to the task of deriving (A.1). Consider, for the moment, the region

$$
D_{L}=\left\{x \in \mathbb{Z}^{2} \mid-12 L \leqq x_{1} \leqq+18 L ;-3 L \leqq x_{2} \leqq+3 L\right\},
$$

which should be regarded as the regions $B_{L}$ and $\mathrm{T}(6 L, 0) B_{L}$ (the translate of $B_{L}$ by $6 L$ units in the $x_{1}$-direction). Suppose that the events $\mathscr{N}_{L}$ and $\mathrm{T}(6 L, 0) \mathscr{N}_{L}$ occur simultaneously. Then, as illustrated in Fig. 5, the two dual paths "almost touch". 
Since we will be concerned with these events in regions larger than $B_{L}$ - where boundary conditions are more favorable for negative events - we may use the $\mu_{B_{L}, w, \Omega}(-)$ and $\left.\mu_{\mathrm{T}(6 L, 0) B_{L}, w, \Omega}(-)\right)$ probabilities of these events to estimate the probability of their simultaneous occurrence.

Given that both of these $\mathcal{N}_{L}$ 's happen, we would like to see the two dual surfaces joined together. To this end, observe that in the region $\left\{0 \leqq x_{1} \leqq+6 L ;-3 L \leqq x_{2}\right.$ $\leqq+3 L\}$ (the center square in Fig. 5) where there are potentially two crossings, there must be a highest and a lowest crossing. We may thus condition on the event that $\sigma_{1}$ is the highest surface and $\sigma_{2}$ the lowest in this region. (These surfaces will not, in general, be disjoint.) The existence of these dual surfaces - in the subset of $\left\{0 \leqq x_{1} \leqq+6 L ;-3 L \leqq x_{2} \leqq+3 L\right\}$ bounded by them - is equivalent to free boundary conditions where they are located. This, of course, favors the formation of additional surfaces. Hence, the probability of a path joining $\sigma_{1}$ and $\sigma_{2}$ (within $\left\{0 \leqq x_{1} \leqq+6 L ;-3 L \leqq x_{2} \leqq+3 L\right\}$ ) with the wired boundary conditions on $D_{L}$ is larger than the probability of finding a path between the locations of $\sigma_{1}$ and $\sigma_{2}$ in the measure which is wired on $\left\{0 \leqq x_{1} \leqq+6 L ;-9 L \leqq x_{2} \leqq+15 L\right\}$ (a translation and rotation of $B_{L}$ ). The latter will be bounded below by the probability of observing (the translation and rotation of) $\mathscr{N}_{L}$ without regard to the details of the locations of the surfaces $\delta_{1}$ and $\sigma_{2}$.

Summing over all possible pairs of surfaces (which represents a partition of the measure), we have that the wired probability in $D_{L}$ of observing a dual path between the lines $x_{1}=-6 L$ and $x_{1}=12 L$ is bounded below by products of probabilities of translations and rotations of $\mathscr{N}_{L}$ in the corresponding $B_{L}$ with wired boundary conditions. Observe that we have so far only used the FKG properties of the FK random cluster measure on a fixed realization $\Omega$.

The above reasoning is summarized, graphically, in Eq. (A.7) below:
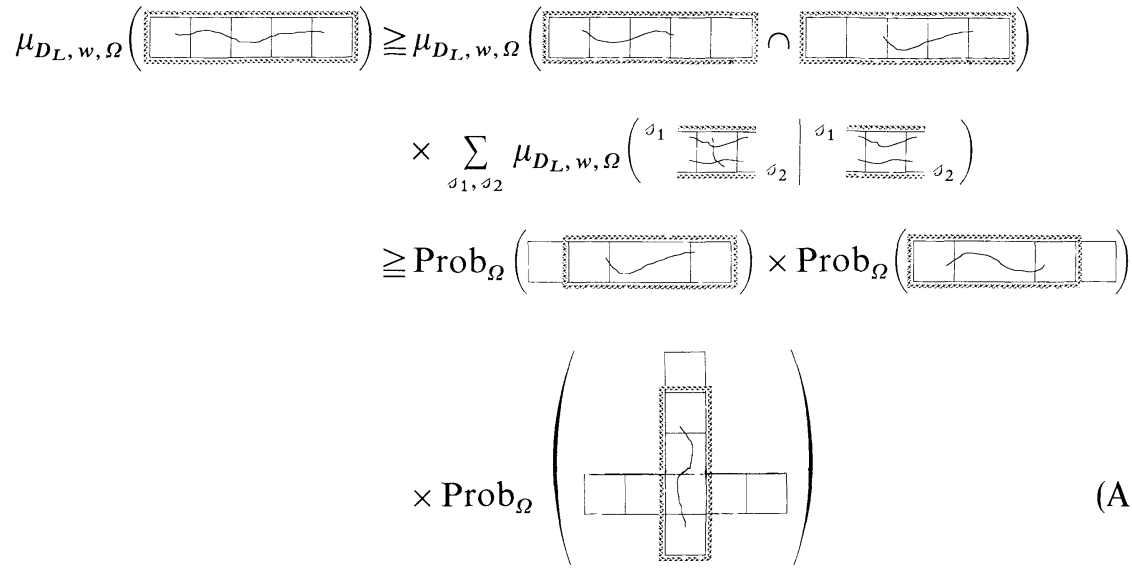

Now, upon quenching, we may exploit the disorder FKG properties for these (negative) events. Then, using translation invariance, we obtain

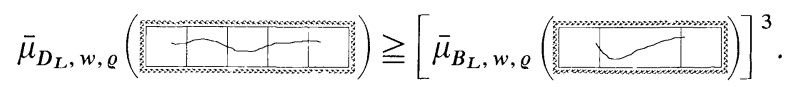



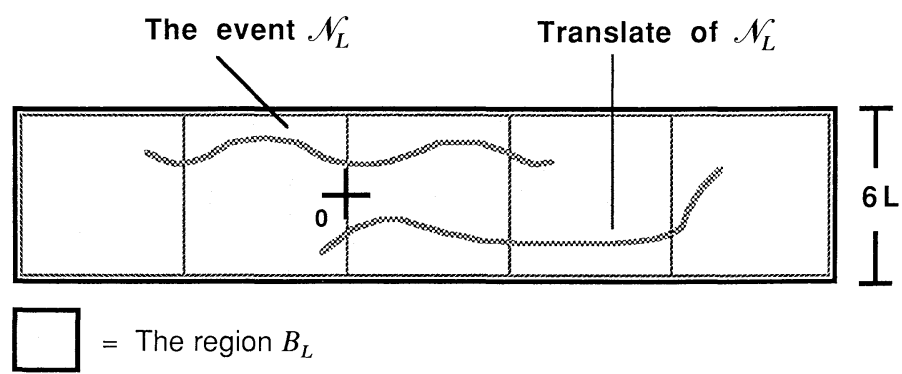

Fig. 5. Two surfaces

Extending the argument across yet another $3 L \times 3 L$ square, one obtains

$$
\bar{\mu}_{E_{L}, w, \varrho}(1+1-1
$$

where $E_{L}$ is the $6 \times 1$ region of scale $L$. Of course, negative events in wired regions are favored by moving the boundary further away, so the event depicted on the lefthand side of (A.9) is less likely than the same surface occurring in the wired $8 \times 1$ region of scale $L$. Hence (A.9) implies (A.1).

The higher dimensional analogue of the argument runs along a similar track. In $d=3$, one can first paste surfaces in the $x_{1}$-direction - which requires five versions of $\mathscr{N}_{L}$ - to obtain a long strip. These strips can be pasted in the $x_{2}$-direction, again five being required to achieve the desired effect. The result, then, in three dimensions is

$$
\bar{\mu}_{B_{L}^{t}, w}\left(\mathscr{N}_{2 L}^{t}\right) \geqq\left[\bar{\mu}_{B_{L}, w}\left(\mathscr{N}_{L}\right)\right]^{25}
$$

or, in general,

$$
\bar{\mu}_{B_{2 L}^{t}, w}\left(\mathscr{N}_{2 L}^{t}\right) \geqq\left[\bar{\mu}_{B_{L}, w}\left(\mathscr{N}_{L}\right)\right]^{5^{d-1}} .
$$

Given this bound, the steps described at the beginning of the proof are identical.

\section{References}

1. Chayes, J.T., Chayes, L., Fisher, D.S., Spencer, T.: Phys. Rev. Lett. 57, 2999 (1986)

2. Harris, A.B.: J. Phys. C 7, 1671 (1974)

3. Mott, N.F.: Commun. Phys. 1, 203 (1976) and Philos. Mag. B 44, 265 (1981)

4. Shankar, R.: Phys. Rev. Lett. 58, 2466 (1987)

5. Newman, K.E., Riedel, E.: Phys. Rev. B 25, 264 (1982)

6. Weinrib, A., Halperin, B.I.: Phys. Rev. B 27, 413 (1983)

7. Chayes, J.T., Chayes, L., Fröhlich, J.: Commun. Math. Phys. 100, 399 (1985)

8. McCoy, B.M., Wu, T.T.: Phys. Rev. 76, 631 (1968)

McCoy, B.M.: Phys. Rev. B 2, 2795 (1970)

9. Shankar, R., Murthy, G.: Phys. Rev. B 36, 536 (1987)

10. Fisher, D.S., Kanter, I.: Unpublished

11. Fortuin, C.M., Kasteleyn, P.W.: Physica 57, 536 (1972)

12. Aizenman, M., Chayes, J.T., Chayes, L., Newman, C.M.: J. Stat. Phys. 50, 1 (1988)

13. Aizenman, M., Chayes, J.T., Chayes, L., Newman, C.M.: J. Phys. A 20, L 313 (1987) 
14. Fortuin, C.M., Kasteleyn, P.W., Ginibre, J.: Commun. Math. Phys. 22, 89 (1971)

15. Harris, T.E.: Proc. Camb. Phil. Soc. 56, 13 (1960)

16. Fortuin, C.M.: Physica 59, 545 (1972)

17. Enter, A.C.D. van, Griffiths, R.B.: Commun. Math. Phys. 90, 319 (1983)

18. Chayes, J.T., Chayes, J.: Critical phenomena, random systems and gauge theories, pp. 1001-1142. In: Proc. of the Les Houches Summer School, Session XLIII. Osterwalder, K., Stora, R. (eds.). Amsterdam: North-Holland 1986

19. Kesten, H.: Private communication

20. Fröhlich, J., Imbrie, J.Z.: Commun. Math. Phys. 96, 145 (1984)

21. Simon, B.: Commun. Math. Phys. 77, 111 (1980)

22. Lieb, E.H.: Commun. Math. Phys. 77, 127 (1980)

23. Aizenman, M., Chayes, J.T., Chayes, L., Fröhlich, J., Russo, L.: Commun. Math. Phys. 92, 19 (1983)

Communicated by A. Jaffe

Received June 27, 1988 
